\title{
EDITORIAL
}

\section{Multidrug-resistant tuberculosis in Eastern Europe: still on the increase?}

\author{
Giovanni Battista Migliori*, Masoud Dara\#, Pierpaolo de Colombani\#, Hans Kluge\# and \\ Mario C. Raviglione
}

$\mathbf{T}$ he recently published World Health Organization (WHO) 2011 Global Report [1] provides important new information on the status of the tuberculosis (TB) epidemic and better estimates are now available on different indicators of ТВ control. These were possible thanks to new methodologies used to measure the disease, producing more precise figures on mortality, number of children orphaned by $\mathrm{TB}$, budget needs and financial gaps. Overall, a clearer picture of $\mathrm{TB}$ in general, and multidrug-resistant (MDR)- and extensively drugresistant (XDR)-TB in particular, is now available to guide the necessary national and international public health response [1-4].

A major response to this is that of the WHO Regional Office for Europe (Copenhagen, Denmark), which has recently prioritised MDR-TB prevention and control by developing a consolidated 5 -yr action plan. The action plan and its resolution were endorsed at the 61st Regional Committee in Baku, Azerbaijan in September 2011. The plan envisages identifying and addressing the causes leading to emergence of drug-resistant $\mathrm{TB}$ in the region [5]. A complex interplay of factors, such as irrational drug policies, and social and clinical determinants (e.g. HIV infection, imprisonment, migration and socioeconomic factors), are driving the emergence and the spread of MDR-TB and XDR-TB $[6,7]$.

Surveillance of drug resistance is a major tool to document the situation at the national level, and the trends in performance of TB care and control efforts, as long as three simple principles are respected: the information collected must be representative of the setting under evaluation; WHO-approved laboratory methods must be used; and the possibility of differentiating new and re-treatment cases must exist [1, 2].

The remarkable study by SKRAHINA et al. [8] published in this issue of the European Respiratory Journal (ERJ) reports the highest ever MDR-TB prevalence in the world within a WHO standard survey performed according to the three simple principles mentioned above. The survey was conducted in Minsk, Belarus: MDR-TB was found in 35.3\% of new cases and in $76.5 \%$ of those previously treated. This means that about

*WHO Collaborating Centre for TB and Lung Diseases, Fondazione S. Maugeri, Care and Research Institute, Tradate, Italy. ${ }^{*}$ World Health Organization, Regional Office for Europe, Copenhagen, Denmark. "Stop TB Dept, World Health Organization, Geneva, Switzerland.

CORRESPONDENCE: G.B. Migliori, WHO Collaborating Centre for Tuberculosis and Lung Diseases, Fondazione S. Maugeri, Care and Research Institute, Tradate Italy. E-mail: giovannibattista.migliori@fsm.it half of the cases diagnosed in that setting harbour MDR-TB strains.

In addition, XDR-TB was found in $14 \%$ of the MDR-TB cases, a proportion much higher than the global average (9.4\%) [2]. Furthermore, the fact that patients $<35$ yrs of age showed twofold higher odds of MDR-TB than those aged $\geqslant 35$ yrs suggests that, in Belarus, transmission of these strains is rampant and recent, confirming the alarming picture already described in some other countries of the former Soviet Union [1].

Importantly, figures obtained within the Minsk routine surveillance system showed a much lower $(26.9 \%$ among new cases and $60.3 \%$ among previously treated cases) prevalence of MDRTB than those the study using international standards revealed [7]. Therefore, a substantial underestimate of the problem was likely to occur in the absence of a proper study.

There are three main questions emerging from the assessment of the results of the Minsk study. 1) Did the study have methodological limitations? 2) What are the reasons behind this alarming situation? 3) What should be done to reverse the situation?

The study has two main limitations, which have been correctly underlined by the authors. First, chronic cases (e.g. the cases failing a supervised re-treatment regimen) were excluded to remove a potential source of bias, as they are likely to be affected by MDR- or XDR-TB strains. This means that, if a bias exists, it is in the direction of underestimating the real prevalence of multidrug resistance. Secondly, the prevalence in the Minsk municipality might be higher than that in rural areas. However, preliminary results from the national survey in Belarus seem to indicate that the prevalence of MDR-TB is as high as in the city of Minsk. In any case, even in the absence of a nationwide survey, it is imperative to conduct a detailed assessment of the factors contributing to the very high level of drug-resistant TB in Minsk, including a molecular epidemiology study to document the transmission of the main strains.

At the moment, the causes are perceived to be multifactorial. The authors report three main reasons: absence of patient support after discharge; long and cumbersome diagnostic procedures; and poor infection control practices [8]. The review of the National TB Programme organised by WHO in October 2011 suggested that these factors are present, and linked to a long and often forced hospitalisation contributing to nosocomial infection and spread of drug-resistant TB. Despite the progress made by the national programme to improve 
services during recent years, the alarming situation of MDR-TB may stem from two decades of an inadequate public health approach to TB control and, particularly, stock-outs of firstand second-line drugs leading to virtual monotherapy during the collapse of the Soviet Union, use of potentially substandard quality drugs, and wrong clinical practices including adding a single drug to a failing regimen [7-11].

Recent evidence from the European Union [7, 9, 11, 12] clearly shows that several deviations from recommended guidelines [3] occur even in higher-income, low-TB incidence countries, creating opportunities for development of highly resistant strains and their spread. Suboptimal infection control practices in health facilities have been described in many settings, exposing patients, visitors and staff to the risk of acquiring infection with MDR- or XDR-TB strains [13].

Clearly, after the description of the highest ever prevalence of MDR-TB, Belarus is at a cross-road. Urgent action is necessary to tackle the problem effectively and quickly. Among solutions to address this alarming situation, the following are mentioned by the authors: the government of Belarus needs to introduce modern technology for the rapid diagnosis of MDR-TB; rational use of hospital care with ensured infection control; and significant scaling up of ambulatory patient support. The estimated high number of MDR-TB cases in need of treatment is a major challenge, as, like in many other countries, Belarus is heavily affected by the current financial crisis. Furthermore, the devaluation of the national currency renders the import of essential TB commodities much more expensive. Therefore, in this public health emergency, external financial support to Belarus is crucial.

Belarus is an ambitious country struggling, like many in that part of the world, to reach economic and public health standards equivalent to those of neighbouring western countries. Commitment by its government to effectively face public health emergencies has been shown in the past few years in a number of circumstances. Prevention and control of MDR-TB requires bold policies and focused interventions. By making the information reported in this issue of the ERJ available to the world and by revealing the need for international support in building the proper response, the Belarus Ministry of Health has shown courage and leadership. There is an urgent need to strengthen primary healthcare, improve models of care and ensure services are patient friendly. It is now the time for donors, technical agencies and those engaged in the fight against TB worldwide to play their part, intervene promptly, and support Belarus technically and financially in this struggle against $T B$, thus saving thousands of lives today and removing the MDR-TB threat to future generations.

\section{STATEMENT OF INTEREST}

None declared.

\section{REFERENCES}

1 World Health Organization. Global Tuberculosis Control 2011. World Health Organization Report 2011, Publication No. WHO/ HTM/TB/2011.16. Geneva, World Health Organization, 2011.

2 Zignol M, van Gemert W, Falzon D, et al. Surveillance of antituberculosis drug resistance in the world: an updated analysis, 2007-2010. Bull World Health Organ 2011; 90: 111D-119D.

3 World Health Organization. Guidelines for the programmatic management of drug-resistant tuberculosis - 2011 update. Publication No. WHO/HTM/TB/2011.6. Geneva, World Health Organization, 2011.

4 Nathanson E, Nunn P, Uplekar M, et al. MDR tuberculosis critical steps for prevention and control. N Engl J Med 2010; 363: 1050-1058.

5 Dara M, Kluge H. Roadmap to Prevent and Combat DrugResistant Tuberculosis. Copenhagen, WHO Regional Office for Europe, 2011.

6 Migliori GB, Sotgiu G, Lange C, et al. Extensively drug-resistant tuberculosis: back to the future. Eur Respir J 2010; 36: 475-477.

7 Abubakar I, Dara M, Manissero D, et al. Tackling the spread of drug-resistant tuberculosis in Europe. Lancet 2012; 379: e21-e13.

8 Skrahina A, Hurevich H, Zalutskaya A, et al. Alarming levels of drug-resistant tuberculosis in Belarus: results of a survey in Minsk. Eur Respir J 2012; 39: 1425-1431.

9 Skrahina A, Zalutskaya A, Sahalchyk E, et al. Determining the true burden of anti-TB drug resistance in Minsk: routine surveillance vs. survey. Int J Tuberc Lung Dis 2011; 15: Suppl. 3, 310S-311S.

10 Sotgiu G, Centis R, D'Ambrosio L, et al. Development of a standardised tool to survey MDR-/XDR-TB case management in Europe. Eur Respir J 2010; 36: 208-211.

11 Sotgiu G, D'Ambrosio L, Centis R, et al. TB and M/XDR-TB infection control in European TB reference centres: the Achilles' heel? Eur Respir J 2011; 38: 1221-1223.

12 Migliori GB, Sotgiu G, Blasi F, et al. Towards the development of EU/EEA Standards for Tuberculosis Care (ESTC). Eur Respir J 2011; 38: 493-495.

13 Dehda K, Migliori GB. The global rise of extensively drug-resistant tuberculosis: is the time to bring back sanitoria now overdue? Lancet 2012; 379: 773-775. 